\title{
Mast Cells Induce Rat Pulmonary Fibroblast Proliferation and Differentiation via Direct Cell-Cell Contact
}

\author{
Zhenshun Cheng*, Jiong Yang and Yanqing Ye \\ Department of Respiratory Medicine, ZhongNan Hospital of WuHan University, \\ WuHan, 430071, China
}

Received August 13, 2012; accepted October 10, 2012

\begin{abstract}
Summary We investigated whether direct cell-cell contact between mast cells (MCs) and pulmonary fibroblasts (PFs) was important for PF proliferation, differentiation, and function, which are critically involved in the development of idiopathic pulmonary fibrosis. MCs and PFs were isolated from male Wistar rats for 3 experimental conditions: (1) PFs alone (Controls); (2) co-culture and contact (CC) between MCs and PFs; and (3) co-culture and no contact (NC) between MCs and PFs. We monitored PF proliferation using growth curves and MTT assays, evaluated PF expression of $\alpha$-smooth muscle actin ( $\alpha$-SMA) by immunocytochemistry, and determined the concentrations of bFGF, TGF- $\beta 1$, and Type I collagen in culture supernatants by ELISA. After 5 days in culture, CC conditions induced a significant increase in PF proliferation compared to the other 2 conditions. No significant difference was observed between the $\mathrm{NC}$ and control conditions. $\mathrm{CC}$ conditions also induced significantly higher PF expressions of $\alpha$-SMA, and bFGF, TGF- $\beta 1$, and Type I collagen production compared to the other 2 conditions. These results suggest that MCs promote both PF proliferation and their differentiation into myofibroblasts via direct cell-cell contact.
\end{abstract}

Key words bFGF, Interstitial pulmonary fibrosis, Mast cells, Pulmonary fibroblasts, TGF- $\beta 1$, Type I collagen.

Idiopathic pulmonary fibrosis is a disease that progresses gradually and may be fatal under certain conditions. Pathologically, IPF is characterized by general interstitial pneumonia with damage to alveolar epithelial cells, fibroblast proliferation, and the deposition of large amounts of extracellular matrix (ECM) in the interstitium (Chilosi et al. 2006). There are currently no effective therapeutic strategies to restrain the development of IPF.

Pulmonary fibroblasts play an important role in the synthesis of collagens and the ECM in the pulmonary interstitium. Thus, understanding the biological behaviors of pulmonary fibroblasts will aid in identifying the mechanisms underlying the formation of pulmonary fibrosis (Lawson et al. 2005). In idiopathic pulmonary fibrosis, there are numerous fibroblast foci in pulmonary connective tissues. These foci are primarily comprised of pulmonary fibroblasts and myofibroblasts, which differentiate from fibroblasts in the pulmonary interstitium. Both fibroblasts and myofibroblasts predominantly secrete Types I and III collagen, the major components of the ECM.

Previous studies of pulmonary fibrosis have emphasized that mast cells (MCs) promoted the formation of tissue fibrosis via their interactions with pulmonary fibroblasts (Garbuzenko et al. 2004). In rats with pulmonary fibrosis, investigators have found that MCs aggregate in areas of pulmonary fibroblast hyperplasia. In IPF, it has been demonstrated that MCs produce large amounts of basic fibroblast growth factor (bFGF), and MC aggregates with high levels of bFGF expression have been found in regions with ECM deposition and proliferation of smooth muscle cells/myofi-

*Corresponding author, e-mail: zhenshun_cheng@126.com

DOI: $10.1508 /$ cytologia. 77.415 
broblasts. These findings suggest that MCs and bFGF are closely related to the proliferation and differentiation of fibroblasts (Inoue et al. 2002).

However, whether direct cell-cell contact between MCs and pulmonary fibroblasts is important for these effects remains uncertain. It has been shown that rat peritoneal MCs could form gap junctional intercellular communications with fibroblasts, which promoted fibroblast proliferation and collagen lattice contraction (Foley et al. 2011). These gap junctional intercellular communications are modulated, in part, by the expression of connexin-43, a part of heterocellular transmembrane protein channels (Pistorio and Ehrlich 2011). Yet, in the context of the development of pulmonary fibrosis and idiopathic pulmonary fibrosis in particular, it remains to be determined if direct cell-cell contacts between MCs and pulmonary fibroblasts is required or if factors secreted by MCs is sufficient for these effects on fibroblasts.

In addition, although previous studies showed that MCs promoted fibroblast proliferation, it was not determined if fibroblast differentiation and function(s) were also affected. Thus, in this study, we investigated whether direct cell-cell contact between MCs and pulmonary fibroblasts was important for pulmonary fibroblast proliferation, differentiation, and function. To this end, pulmonary fibroblasts were cultured either with or without direct contact with MCs. Our results suggest that MCs promote pulmonary fibroblast proliferation and differentiation via their secretion of growth factors, and these effects are potentiated by direct cell-cell contact.

Materials and methods

Animals

Ten adult male Wistar rats (2-3-months old; 180-250g) were purchased from the Experimental Animal Center of the Center for Disease Control in Hubei Province. Approval for use of animals in experiments was provided by the ethical review board of ZhongNan Hospital, WuHan University.

\section{Isolation and culture of primary pulmonary fibroblasts}

Rats were anesthetized by intraperitoneal injection of urethane $(1 \mathrm{~g} / \mathrm{kg})$ and then immersed in disinfectant. Thoracotomy and laparotomy were performed in an aseptic environment and rats were perfused transcardially with $\mathrm{Ca}^{2+}$ and $\mathrm{Mg}^{2+}$ free PBS through the right ventricle. The abdominal aorta was opened and artificial ventilation was provided via endotracheal intubation. When the lungs became grey, they were lavaged with $\mathrm{Ca}^{2+}$ and $\mathrm{Mg}^{2+}$ free PBS $(50 \mathrm{ml})$ through the endotracheal tube. The lungs were harvested aseptically and cut into pieces. These pieces were washed with distilled water containing penicillin $(1: 1000)$ and streptomycin $(1: 400)$ and then 3 times with DMEM. The lung pieces were digested in DMEM containing $3 \mathrm{mg} / \mathrm{ml}$ trypsin and $2.5 \mathrm{mg} / \mathrm{ml}$ DNase I 3 times at $37^{\circ} \mathrm{C}$ in a $5 \% \mathrm{CO}_{2}$ atmosphere (fresh solution was prepared for each digestion). After each round of digestion, the supernatant was collected and mixed with an equal volume of DMEM containing $15 \%$ newborn calf serum (NBCS). This mixture was filtered through a mesh and the solution was centrifuged at $1000 \mathrm{rpm}$ at $25^{\circ} \mathrm{C}$ for $8 \mathrm{~min}$. Cells were re-suspended in DMEM containing $10 \%$ NBCS and then seeded into flasks followed by incubation at $37^{\circ} \mathrm{C}$ a in a $5 \% \mathrm{CO}_{2}$ atmosphere for $24 \mathrm{~h}$. Adherent cells were pulmonary fibroblasts.

\section{Identification of primary pulmonary fibroblasts}

Isolated cells' morphologies were observed under an inverted phase contrast microscope. For identification by immunohistochemistry, cover slips were placed into flasks and lung fibroblasts were allowed to adhere to the cover slips. Cell-coated cover slips were fixed in acetone for $10 \mathrm{~min}$ at $4^{\circ} \mathrm{C}$. Immunohistochemistry used a streptavidin-peroxidase method according to the manufacturer's instructions. The cover slips were washed in PBS and then treated with $3 \% \mathrm{H}_{2} \mathrm{O}_{2}$ in methanol to inactivate endogenous peroxidase. Subsequently, cover slips were blocked with normal goat 

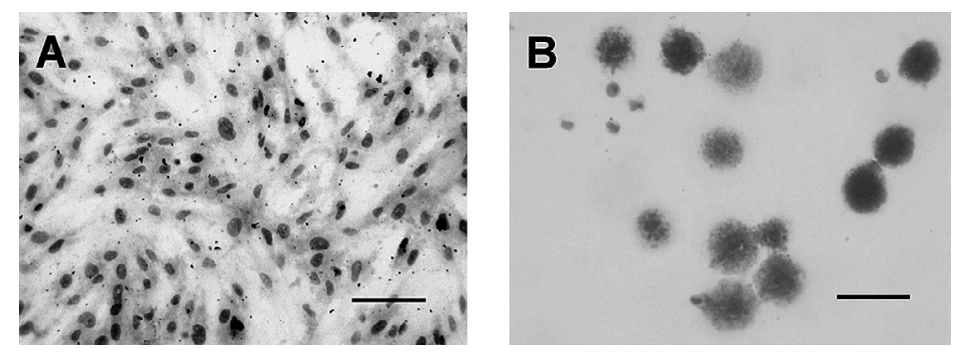

Fig. S1. Validation of primary cells used in this study. (1A) Pulmonary fibroblasts were immunostained with anti-vimentin antibody and developed using DAB assay. (1B) Isolated mast cells were stained with Toluidine Blue and observed under a light microscope. Scale bar $=20 \mu \mathrm{m}$.

serum and then treated with primary antibody (anti-vimentin monoclonal antibody) and secondary antibody (biotin-conjugated goat anti-mouse IgG). Subsequently, cover slips were incubated with HRP conjugated streptavidin (S-A/HRP) followed by visualization at room temperature with DAB. Counterstaining was with hematoxylin followed by dehydration and mounting with neutral gum. Cells were observed under a light microscope (Fig. S1A).

\section{Isolation and purification of rat peritoneal mast cells}

Rats were anesthetized with ether and immersed in a disinfectant, and then euthanized by carotid artery exsanguinations. The abdomen was sterilized with an iodophor, after which $10 \mathrm{ml}$ of D-Hanks solution was injected intraperitoneally. The abdomen was massaged for 2-3 min followed by laparotomy done in an aseptic environment. Peritoneal fluid was collected with a syringe and centrifuged at $1000 \mathrm{rpm}$ for $10 \mathrm{~min}$ at $4^{\circ} \mathrm{C}$. The supernatant was removed and cells were re-suspended in buffer. We purified mast cells from peritoneal cells using Ficoll (Sigma, St. Louis, MO) (Cabado et al. 1993). Briefly, a 40\% Ficoll solution (density $1.120 \mathrm{~g} / \mathrm{L}$ ) was diluted gradually with Ficoll solution used for lymphocyte isolation (density $1.077 \mathrm{~g} / \mathrm{L}$ ) to reduce the density to $1.080 \mathrm{~g} / \mathrm{L}$. Then, $10 \mathrm{ml}$ of this Ficoll solution was placed in a centrifuge tube and $1 \mathrm{ml}$ of a peritoneal exudate cell suspension was carefully layered over the Ficoll layer. After centrifugation at $4000 \mathrm{rpm}$ for $15 \mathrm{~min}$ at $4^{\circ} \mathrm{C}$, the supernatant was removed, and cells at the bottom of the tube were collected. To estimate the purity of the mast cells, the collected cells were re-suspended in RPMI-1640, smeared on a glass slide, and stained with toluidine blue. Mast cell morphology was observed under a light microscope (Fig. S1B).

Mast cell purity was $>95 \%$ in cell suspension.

\section{Experimental cell culture groups}

Three different groups of cells were cultured: (1) pulmonary fibroblasts alone (control group); (2) contact and co-culture (CC group): mast cells (MCs) were co-cultured with pulmonary fibroblasts with direct cell-cell contact between the cell types; (3) non-contact and co-culture group (NC group): MCs were co-cultured with pulmonary fibroblasts, but there was no contact between these cells.

\section{Cell culture}

Cells were harvested at passage 3 in 24-well plates with and without round cover slips. Cells in logarithmic growth phase were digested with $0.25 \%$ trypsin at room temperature for $5 \mathrm{~min}$, harvested, and then washed in HBSS to remove trypsin. These cells were suspended in $1 \mathrm{ml}$ of culture medium. A hemacytometer was used to count cells and determine cell density.

For the NC group, a Transwell insert (6.5 mm in diameter; Costar, USA) was placed into each well to assure that the 2 cell types did not come into contact. Pulmonary fibroblasts at passage 3 
were collected by digestion, re-suspended in freshly prepared culture medium at a density of $1 \times 10^{5}$ / $\mathrm{ml}$, and this cell suspension was added to plates $\left(1 \times 10^{5}\right.$ cells/well). Two days later, the culture medium was refreshed. Then, $1 \mathrm{ml}$ of an MC suspension was added either to a well for direct cell-cell contact (CC group) or to a well with a Transwell insert (NC group). For the control group, pulmonary fibroblasts were cultured without MCs. All cells were then cultured for $5 \mathrm{~d}$.

\section{Morphology and growth of pulmonary fibroblasts}

Cells were maintained in 24-well plates. Pulmonary fibroblasts were cultured for $24 \mathrm{~h}$, with or without co-culture with MCs, after which pulmonary fibroblast morphology was observed under an inverted phase contrast microscope. At the same time, cells from 3 wells were collected and the number of pulmonary fibroblasts was counted. Counting was performed 3 times with averaging each day for 5 consecutive days to generate pulmonary fibroblast growth curves.

\section{MTT assay to determine MC effects on pulmonary fibroblast proliferation}

Cells were maintained in 24-well plates for $72 \mathrm{~h}$. Then, cells were collected from 3 wells for each experimental group by digestion with $0.25 \%$ trypsin at room temperature for $5 \mathrm{~min}$. Cells were washed 3 times with HBSS to remove trypsin, re-suspended in $1 \mathrm{ml}$ of culture medium, and $200 \mu \mathrm{l}$ of this suspension was transferred to a $96-w e l l$ plate.

Cells were maintained at $37^{\circ} \mathrm{C}$ in a $5 \% \mathrm{CO}_{2}$ atmosphere for $24 \mathrm{~h}$ to allow cell adhesion to the microtest plate wall. The supernatant was carefully removed and $80 \mu \mathrm{l}$ of freshly prepared RPMI 1640 was added. Cells in each well were incubated with $20 \mu \mathrm{l}$ of MTT solution $(5 \mathrm{mg} / \mathrm{ml})$ for $4 \mathrm{~h}$. The supernatant was then removed and $100 \mu \mathrm{l}$ of DMSO was added to each well followed by shaking for $10 \mathrm{~min}$ to dissolve the crystals. Absorbance was measured at $490 \mathrm{~nm}$ with a microplate reader. Results were reported as arbitrary optical density (OD) units.

\section{Detection of $\alpha$-SMA by immunocytochemistry}

Cells in logarithmic growth phase $\left(1 \times 10^{5} / \mathrm{ml}\right)$ were seeded on sterile cover slips in a 24 -well plate followed by incubation for $24 \mathrm{~h}$. When cell confluence reached $90 \%$, the medium was removed and fresh RPMI1640 medium containing 15\% calf serum was added. Then, $1 \mathrm{ml}$ of MC suspension $\left(1 \times 10^{4} / \mathrm{ml}\right)$ was added either to each well for direct cell-cell contact (CC group) or into a well with a Transwell insert (NC group). For the control group, fibroblasts alone were maintained. After incubation for $48 \mathrm{~h}, \alpha$-SMA expression was determined by immunocytochemistry using an anti- $\alpha$-SMA mAb according to the supplier's recommendations (R\&D Systems, Minneapolis, MN).

\section{ELISAs for bFGF, TGF- $\beta 1$, and Type I collagen}

MCs were cultured with pulmonary fibroblasts for $5 \mathrm{~d}$. Then, supernatants were collected and the amounts of bFGF, TGF- $\beta 1$, and Type I collagen were determined with ELISA kits according to the manufacturer's instructions (R\&D Systems, Minneapolis, MN).

\section{Statistical analysis}

For fibroblast growth curves, cell numbers were plotted as means \pm standard errors of the mean (SEM's) each day for 5 days. Comparisons among experimental cell culture groups were by repeated ANOVA measurements. Results for fibroblast proliferation, fibroblast expression of $\alpha$-SMA, and ELISA results for TGF- $\beta 1, \mathrm{bFGF}$, and collagen I are given as means \pm SEM's by experimental group. Groups were compared using one-way ANOVA with a Bonferroni adjustment for post-hoc pair-wise comparisons. $p<0.05$ was considered significant. An adjusted significance level of 0.0167 $(0.05 / 3)$ was applied for the post-hoc multiple comparisons. Statistical analyses used SPSS 15.0 statistics software (SPSS Inc., Chicago, IL, USA). 
(A)

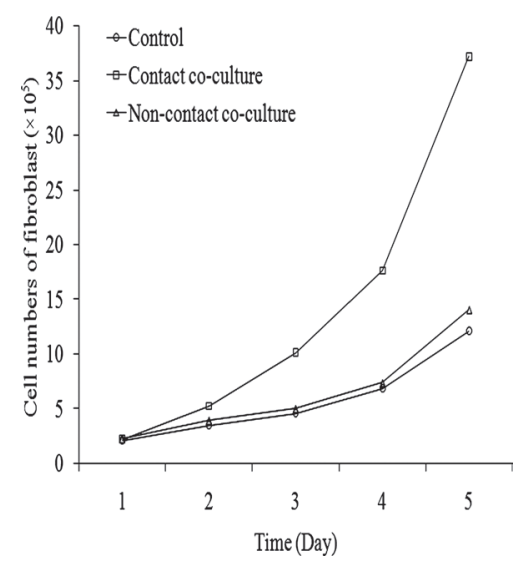

(B)

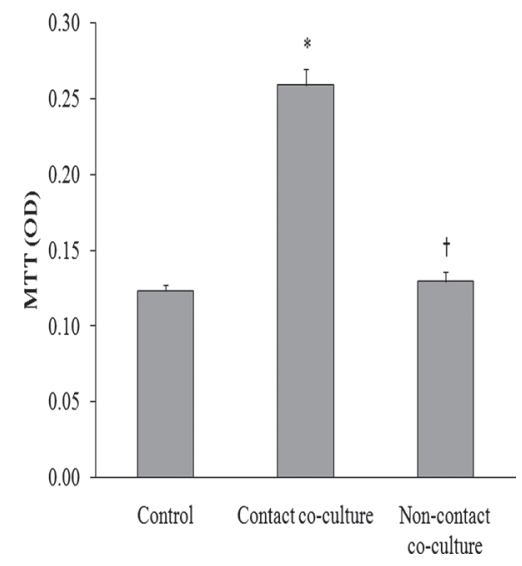

Fig. 1. Pulmonary fibroblast proliferation. The proliferation of pulmonary fibroblasts in control, contact co-culture (CC), and non-contact (NC) co-culture groups were determined by (A) changes in fibroblast cell numbers for $5 \mathrm{~d}$, and (B) MTT assays after $5 \mathrm{~d}$ of culture. Results are means \pm SEM's $(n=3$ / group). In (A), the change in fibroblast cell numbers by day was significantly different among the 3 groups by repeated ANOVA measurements $(p<0.05)$ In $(\mathrm{B})^{*}$ indicates significantly different as compared with the control group $(p<0.0167)$; $\dagger$ indicates significantly different as compared with the contact co-culture group $(p<0.0167)$.

\section{Results}

\section{Morphology of pulmonary fibroblasts}

In the CC group, pulmonary fibroblasts were monolayer-like after culture for $1-2 \mathrm{~d}$. Fibroblasts then grew into multiple layers with overlapping, and had network-like growth at $3 \mathrm{~d}$ of culture. In the NC and control groups, pulmonary fibroblasts grew as monolayers.

\section{MC effects on pulmonary fibroblast proliferation}

Pulmonary fibroblast growth was followed for $5 \mathrm{~d}$ with and without MC co-culture (Fig. 1A). In the $\mathrm{CC}$ group, the numbers of pulmonary fibroblasts were significantly increased when compared with the $\mathrm{NC}$ and control groups. For all groups, the numbers of pulmonary fibroblasts peaked at $5 \mathrm{~d}$ of culture: $\mathrm{CC}$ group $=3.7 \times 10^{6} \pm 0.83 \mathrm{cells} / \mathrm{well}$; $\mathrm{NC}$ group $=1.4 \times 10^{6} \pm 0.32 \mathrm{cell} / \mathrm{s} / \mathrm{well}$; Control group $=1.1 \times 10^{6} \pm 0.23$ cells/well. At $5 \mathrm{~d}$, the number of pulmonary fibroblasts in the $\mathrm{CC}$ group was significantly higher than those in the control and NC groups $(p<0.05)$. In addition, the number of pulmonary fibroblasts in the NC group was only slightly higher than that in the control group.

After $5 \mathrm{~d}$ of culture, MTT assays were also used to assess fibroblast proliferation (Fig. 1B). The measured absorbance (OD units) for the CC group was significantly higher than for the control and NC groups $(p<0.05)$. This suggested that pulmonary fibroblasts were more actively proliferating in the CC group than in the control and NC groups. Again, no marked difference was found between the control and $\mathrm{NC}$ groups $(p>0.05)$.

\section{Detection of $\alpha-S M A$ in pulmonary fibroblasts}

Pulmonary fibroblast expression of $\alpha$-SMA with and without MC co-culture was detected by immunocytochemistry. Representative photomicrographs of $\alpha$-SMA expression are shown in Fig. 2 for the (A) Control, (B) CC, and (C) NC groups (darker color indicates more intense staining). In the control group, only a few cells were positive for $\alpha$-SMA. In the CC group, large numbers of cells exhibited positive staining for $\alpha$-SMA in the cytoplasm. Figure 2D shows that the integrated 

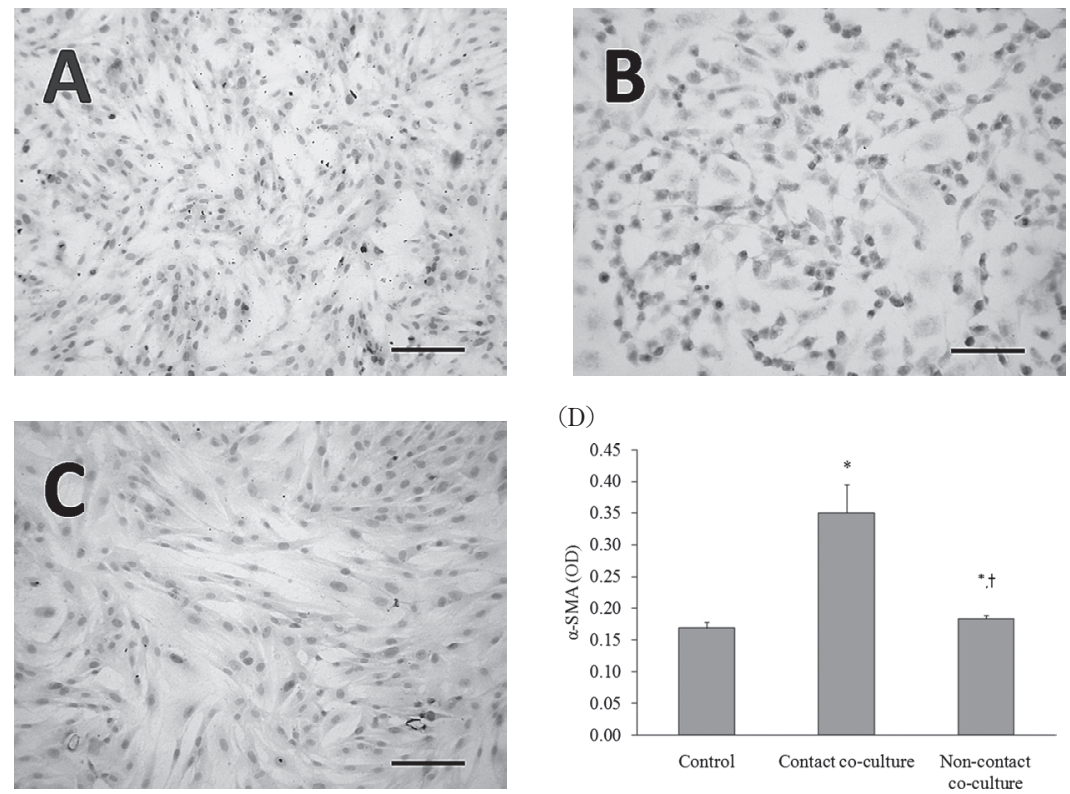

(D)

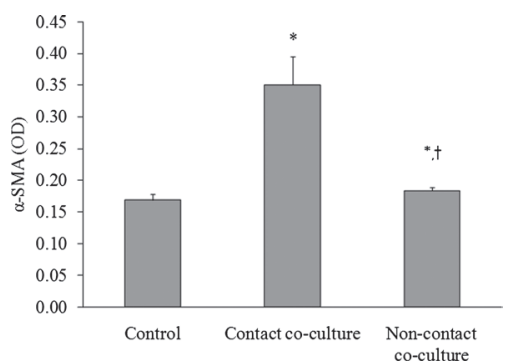

Fig. 2. Pulmonary fibroblast expression of $\alpha$-SMA. Representative photomicrographs showing $\alpha$-SMA staining results for (A) control, (B) contact co-culture, and (C) non-contact co-culture groups after culture for $48 \mathrm{~h}$. (D) Integrated optical density (OD) readings for $\alpha$-SMA staining. Results are means \pm SEM ( $n=3$ /group). $\alpha$-SMA expression levels were: Controls $=0.17 \pm 0.01$; Contact co-culture $=0.35 \pm 0.04$; and Non-contact co-culture $=0.18 \pm 0.01$. $*$ indicates significantly different as compared with the control group $(p<0.0167) ;{ }^{\dagger}$ indicates significantly different as compared with the contact co-culture group $(p<0.0167)$.

(A)

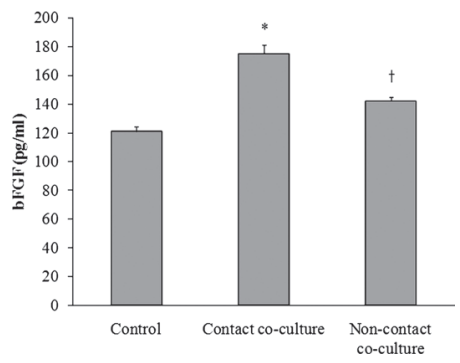

(B)

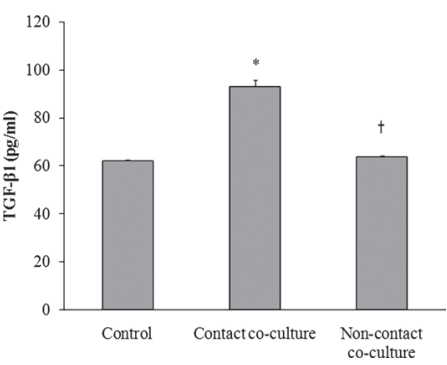

(C)

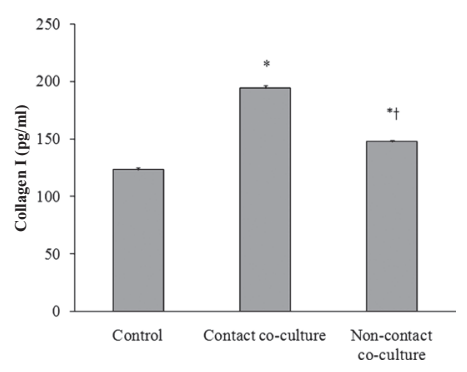

Fig. 3 Cell culture production of bFGF, TGF- $\beta 1$, and Type I Collagen. ELISA's were used to determine supernatant concentrations of (A) bFGF, (B) TGF- $\beta 1$, and (C) Type I Collagen for control, contact coculture, and non-contact co-culture groups after culture for $5 \mathrm{~d}$. Results are means \pm SEM's $(n=3$ / group). * indicates significantly different as compared with the control group $(p<0.0167) ;{ }^{\dagger}$ indicates significantly different as compared with the contact co-culture group $(p<0.0167)$.

optical density (IOD) results for $\alpha$-SMA expression in the CC group were dramatically higher than the results for the $\mathrm{NC}$ and control groups. This suggested that numerous pulmonary fibroblasts in the $\mathrm{CC}$ group had differentiated into myofibroblasts. $\alpha$-SMA expression was comparable between the $\mathrm{NC}$ and $\mathrm{CC}$ groups.

\section{Cell culture production of bFGF, TGFB1, and Type I collagen}

As shown in Fig. 3, ELISAs were used to determine the supernatant concentrations of (A) 
bFGF, (B) TGF $\beta 1$, and (C) Type I collagen after $5 \mathrm{~d}$ of pulmonary fibroblast culture with and with $\mathrm{MC}$ co-culture. The bFGF concentration in the CC group (Fig. 3A) was significantly increased compared to the $\mathrm{NC}$ and control groups $(p<0.05)$, suggesting that bFGF secretion had increased in the CC group. Although the bFGF level in the NC group appeared to be higher than that in the control group, this was not significantly different $(p>0.05)$

The TGF $\beta 1$ level (Fig. 3B) in the CC group was also markedly higher than those in the NC and control groups $(p<0.05)$. Again, although the TGF $\beta 1$ level in the NC group was apparently higher than that in the control group, this was not significantly different $(p>0.05)$.

For Type I collagen (Fig. 3C), the level in the CC group was also markedly increased compared to the NC and control groups $(p<0.05)$. This suggested that the secretion of Type I collagen by fibroblasts and myofibroblasts in the CC group had increased. Type I collagen levels were not significantly different between the NC and control groups $(p>0.05)$.

\section{Discussion}

Our results showed that co-culturing pulmonary fibroblasts with mast cells (MCs) promoted the proliferation of pulmonary fibroblasts. As compared to the control (fibroblasts alone) and no cell contact (NC) groups, the proliferation of pulmonary fibroblasts was significantly increased in our co-culture with contact (CC) group. These results are in agreement with previous studies in which MCs promoted fibroblast proliferation in vitro (Garbuzenko et al. 2004, Foley et al. 2011). In addition, our co-culture conditions promoted fibroblast differentiation into myelofibroblasts (increased $\alpha$-SMA expression), and induced increased production of growth factors (bFGF, TGF $\beta 1$ ) and Type I collagen. Thus, our results suggest MCs facilitate several fibrosis-promoting activities of fibroblasts via direct cell-cell contacts.

However, although this preliminary investigation suggests the importance of cell-cell contact between MCs and fibroblasts, the particular mechanisms that may be involved are considerable, as MCs can affect fibroblast behavior via a number of mechanisms. MCs play important roles in several types of fibrosis and some MCs can synthesize and secrete numerous active mediators (histamine, chymotrypsin, trypsin, heparin) and cytokines (TGF- $\beta 1$, TNF- $\alpha$, fibroblast growth factor). These factors can stimulate the proliferation of fibroblasts and their over-deposition of ECM, which plays an important role in tissue fibrosis (Zhong et al. 2001, Liu et al. 2003).

With regard to fibrosis, an important effect of MCs on pulmonary fibroblasts is promoting their differentiation into myofibroblasts, as evidenced by $\alpha$-SMA expression detected by immunocytochemistry. Our results showed that only a few pulmonary fibroblasts were positive for $\alpha$-SMA when cultured alone, but that large numbers of cells in the CC group were positive for $\alpha$-SMA. The IOD readings for $\alpha$-SMA expression in the CC group were significantly higher than those in the control and NC groups (Fig. 2; $p<0.05$ ). This suggested that co-culture with direct contact between fibroblasts and MCs facilitated the differentiation of pulmonary fibroblasts into myofibroblasts. However, this will require further investigation, particularly with regard to human pulmonary fibrosis. Human lung fibroblasts are heterogeneous, although their differentiation appears to be under the control of a TGF-b signaling pathway (Gu et al. 2007, Pechkovsky et al. 2010). TGF- $\beta$ induces Smad signal pathway mediated $\alpha$-SMA gene expression, which is a key marker of myofibroblast differentiation (Gu et al. 2007).

The fibrosis promoting effects of MCs was also supported by our results for Type I collagen in culture supernatants (Fig. 3B). Type I collagen is a major component of the ECM and is synthesized by fibroblasts and myofibroblasts, particularly myofibroblasts. In our CC group, the Type I collagen level was significantly higher than that in the control group. Again, however, the detailed mechanisms for this activity will have to be investigated. As examples, previous studies have shown that MC tryptase activity (Cairns and Walls 1997, Akers et al. 2000) and matrix metallopro- 
teinase (MMP) activity of MCs (Margulis et al. 2009) could promote human lung fibroblasts to produce Type I collagen. TGF- $\beta$ is another strong inducer for collagen production in human fetal lung fibroblasts (Krupsky et al. 1996).

Our results also showed that the amounts of bFGF and TGF- $\beta 1$ in the culture supernatants of our CC group were dramatically increased as compared with the control and NC groups. This indicated that co-culturing MCs and fibroblasts increased the secretion of both of these cytokines by fibroblasts and that direct contact between these cell types potentiated this effect. bFGF promotes the growth of fibroblasts, vascular endothelial cells, smooth muscle cells, and neurons. Regarding a correlation between bFGF and pulmonary disease, studies have confirmed that bFGF plays pivotal roles in the growth of lung cancer cells and in angiogenesis observed in lung cancer (Ye and Xie 2006, Grozio et al. 2007). bFGF promotes vascular structure remodeling in COPD patients and pulmonary heart disease patients, and promotes airway remodeling in asthma patients (Ward et al. 2004, Ye and Xie 2006). In idiopathic pulmonary fibrosis, myofibroblasts and pulmonary fibroblasts play critical roles. In addition, numerous MCs with high expression levels of bFGF are found to be aggregated in regions of ECM deposition and smooth muscle cells/myofibroblasts hyperplasia. This suggests that bFGF and MCs are associated with the proliferation and differentiation of pulmonary fibroblasts (Grozio et al. 2007).

It is well known that TGF- $\beta 1$ is one critical factor that causes idiopathic pulmonary fibrosis (Ask et al. 2006). TGF- $\beta 1$ is a powerful activator of connective tissue synthesis and fibroblast proliferation in the lung, and a critical paracrine signal for the development of pulmonary fibrosis (Xiao et al. 2012). TGF- $\beta 1$ induces fibroblast growth factor 2 (FGF-2) secreted by alveolar epithelial cells, which promotes fibroblast proliferation and fibrotic activation through the ERK kinase pathway (Xiao et al. 2012). TGF- $\beta 1$ has also been shown to regulate the autocrine induction of bFGF in primary human lung fibroblasts (Finlay et al. 2000), and TGF- $\beta 1$ markedly increased the protein expression of the fibroblast growth factor (FGF) receptors FGFR-1 (Flg) and FGFR-2 (Bek) in a time- and dose-dependent manner in human lung fibroblasts (Thannickal et al. 1998).

Taken together, the increased expression of $\alpha$-SMA on pulmonary fibroblasts, and bFGF, TGF- $\beta 1$, and Type I collagen levels in culture supernatants under contact conditions might be mediated by TGF- $\beta 1$ secreted either by mast cells or fibroblasts during their co-culture. TGF- $\beta 1$ might induce bFGF secretion by pulmonary fibroblast in an autocrine manner, which would subsequently induce cell proliferation and differentiation. However, TGF- $\beta 1$ might also directly induce the production of Type I collagen and the expression of $\alpha$-SMA on pulmonary fibroblasts (Krupsky et al. 1996, Grozio et al. 2007). These details will require further investigation.

The major limitation of this study was that our results only suggest that direct cell-cell contact between MCs and fibroblasts was essential for the effects reported here. However, additional ultrastructural studies will be necessary to confirm direct intercellular connections between MCs and fibroblasts under these conditions. Alternatively, blocking cell-cell contacts, such as with an antibody directed against connexin-43 (Pistorio and Ehrlich 2011), will more clearly establish the importance of these intercellular communications.

In summary, as compared with our NC and control groups, co-culture of MCs and pulmonary fibroblasts with direct contact between these 2 cell types promoted the proliferation and differentiation of pulmonary fibroblasts. In addition, co-culture of MCs and pulmonary fibroblasts with direct contact markedly increased the amounts of bFGF and TGF- $\beta 1$ that were produced. Although this was a only a preliminary investigation, we speculate that MCs can affect the proliferation and differentiation of pulmonary fibroblast via promoting the secretion of cytokines, including bFGF and TGF- $\beta 1$, and that direct cell-cell contact between these cell types potentiates these effects. Thus, mast cells may play a crucial role in the pathogenesis of pulmonary fibrotic diseases, including idiopathic pulmonary fibrosis. 


\section{References}

Akers, I. A., Parsons, M., Hill, M. R., Hollenberg, M. D., Sanjar, S., Laurent, G. J. and McAnulty, R. J. 2000. Mast cell tryptase stimulates human lung fibroblast proliferation via protease-activated receptor-2. Am. J. Physiol. Lung. Cell Mol. Physiol. 278: L193-201

Ask, K., Martin, G. E., Kolb, M. and Gauldie, J. 2006. Targeting genes for treatment in idiopathic pulmonary fibrosis: Challenges and opportunities, promises and pitfalls. Proc. Am. Thorac. Soc. 3: 389-393

Cabado, A. G., Vieytes, M. R. and Botana, L. M. 1993. Rat pleural and peritoneal mast cells stimulated at different levels: difference in and influence of purification media. Int. Arch. Allergy Immunol. 100: 234-239

Cairns, J. A. and Walls, F. 1997. Mast cell tryptase stimulates the synthesis of type I collagen in human lung fibroblasts. J. Clin. Invest. 99: 1313-1321

Chilosi, M., Zamò, A., Doglioni, C., Reghellin, D., Lestani, M., Montagna, L., Pedron, S., Ennas, M. G., Cancellieri, A., Murer, B. and Poletti, V. 2006. Migratory marker expression in fibroblast foci of idiopathic pulmonary fibrosis. Respir. Res. 7: 95

Finlay, G. A., Thannickal, V. J., Fanburg, B. L. and Paulson, K. E. 2000. Transforming growth factor- $\beta 1$-induced activation of the ERK pathway/activator protein-1 in human lung fibroblasts requires the autocrine induction of basic fibroblast growth factor. J. Biol. Chem. 275: 27650-27656

Foley, T. T., Saggers, G. C., Moyer, K. E. and Ehrlich, H. P. 2011. Rat mast cells enhance fibroblast proliferation and fibroblast-populated collagen lattice contraction through gap junctional intercellular communications. Plast Reconstr. Surg. 127: 1478-1486

Garbuzenko, E., Berkman, N., Puxeddu, I., Kramer, M., Nagler, A. and Levi-Schaffer, F. 2004. Mast cells induce activation of human lung fibroblasts in vitro. Exp. Lung Res. 30: 705-721

Grozio, A., Catassi, A. Cavalieri, Z., Paleari, L., Cesario, A. and Russo P. 2007. Nicotine, lung and cancer. Anticancer Agents Med. Chem. 7: 461-466

Gu, L., Zhu, Y. J., Yang, X., Guo, Z. J., Xu, W. B. and Tian, X. L. 2007. Effect of TGF- $\beta /$ Smad signaling pathway on lung myofibroblast differentiation. Acta Pharmacol. Sin. 28: 382-391

Inoue, Y., King, T. E. Jr, Barker, E., Daniloff, E. and Newman, L. S. 2002. Basic fibroblast growth factor and its receptors in idiopathic pulmonary fibrosis and lymphangioleiomyomatosis. Am. J. Respir. Crit. Care Med. 166: 765-773

Krupsky, M., Fine, A., Kuang, P. P., Berk, J. L. and Goldstein, R. H. 1996. Regulation of type I collagen production by insulin and transforming growth factor-beta in human lung fibroblasts. Connect. Tissue. Res. 34: 53-62

Lawson, W. E., Polosukhin, V. V., Zoia, O., Stathopoulos, G. T., Han, W., Plieth. D., Loyd, J. E., Neilson, E. G. and Blackwell, T. S. 2005. Characterization of fibroblast-specific protein 1 in pulmonary fibrosis. Am. J. Respir. Crit. Care Med. 8: 899-907

Liu, X. M., Zhu, Z. H. and Deng, A. G. 2003. Related research development of stem cell factor and mast cells. Foreign Med. Sci. (Pathophysiology Clin. Med.) 23: 481-483 (in Chinese)

Margulis, A., Nocka, K. H., Wood, N. L., Wolf, S. F., Goldman, S. J. and Kasaian, M. T. 2009. MMP dependence of fibroblast contraction and collagen production induced by human mast cell activation in a three-dimensional collagen lattice. Am. J. Physiol. Lung Cell Mol. Physiol. 296: L236-247

Pechkovsky, D. V., Hackett, T. L., An, S. S., Shaheen, F., Murray, L. A. and Knight, D. A. 2010. Human lung parenchyma but not proximal bronchi produces fibroblasts with enhanced TGF- $\beta$ signaling and $\alpha$-SMA expression. Am. J. Respir. Cell Mol. Biol. 43: 641-651

Pistorio, A. L. and Ehrlich, H. P. 2011. Modulatory effects of connexin-43 expression on gap junction intercellular communications with mast cells and fibroblasts. J. Cell. Biochem. 112: 1441-1449

Thannickal, V. J., Aldweib, K. D., Rajan, T. and Fanburg, B. L. 1998. Upregulated expression of fibroblast growth factor (FGF) receptors by transforming growth factor- $\beta 1$ (TGF- $\beta 1$ ) mediates enhanced mitogenic responses to FGFs in cultured human lung fibroblasts. Biochem. Biophys. Res. Commun. 251: 437-441

Ward, J. E., Gould, H., Harris, T., Bonacci, J. V. and Stewart, A. G. 2004. PPAR gamma ligands, 15-deoxy-delta12,14prostaglandin $\mathrm{J} 2$ and rosiglitazone regulate human cultured airway smooth muscle proliferation through different mechanisms. Br. J. Pharmacol. 141: 517-525

Xiao, L., Du, Y., Shen, Y., He, Y., Zhao, H. and Li, Z. 2012. TGF-beta 1 induced fibroblast proliferation is mediated by the FGF-2/ERK pathway. Front Biosci. 17: 2667-2674

Ye, A. X. and Xie, Z. F. 2006. Progress on basic fibroblast growth factor in pulmonary disease. Int. J. Respir. 26: 499-502 (in Chinese)

Zhong, Y., Ding, K. and Ye, W. 2001. The relation between expression of basic fibroblast growth factor and mast cells in pterygium. Chin. J. Ophthalmol. 37: 455-457 (in Chinese) 